\title{
Comparison of quasistatic to impact mechanical properties of multiwall carbon nanotube/polycarbonate composites
}

\author{
Paul A. Brühwiler ${ }^{\text {a) }}$ \\ Empa, Swiss Federal Laboratories for Materials Testing and Research, \\ CH-9014 St. Gallen, Switzerland; and Department of Physics and Materials Science, \\ Uppsala University, SE-751 21 Uppsala, Sweden \\ Michel Barbezat and Adly Necola \\ Empa, CH-8600 Dübendorf, Switzerland \\ Doug J. Kohls \\ Department of Chemical and Materials Engineering, University of Cincinnati, \\ Cincinnati, Ohio 45221-0012 \\ Oliver Bunk \\ Paul Scherrer Institute, Swiss Light Source, CH-5232 Villigen PSI, Switzerland \\ Dale W. Schaefer ${ }^{\text {) }}$ \\ Department of Chemical and Materials Engineering, University of Cincinnati, \\ Cincinnati, Ohio 45221-0012 \\ Petra Pötschke \\ Leibniz Institute of Polymer Research Dresden, Department of Polymer Reactions and Blends, \\ D-01069 Dresden, Germany
}

(Received 17 February 2010; accepted 2 March 2010)

We report the quasistatic tensile and impact penetration properties (falling dart test) of injection-molded polycarbonate samples, as a function of multiwall carbon nanotube (MWNT) concentration $(0.0-2.5 \%)$. The MWNT were incorporated by dilution of a commercial MWNT/polycarbonate masterbatch. The stiffness and quasistatic yield strength of the composites increased approximately linearly with MWNT concentration in all measurements. The energy absorbed in fracture was, however, a negative function of the MWNT concentration, and exhibited different dependencies in quasistatic and impact tests. Small-angle x-ray scattering (SAXS) showed that the dispersion of the MWNT was similar at all concentrations. The negative effects on energy absorption are attributed to agglomerates remaining in the samples, which were observed in optical microscopy and SAXS. Overall, there was a good correspondence between static and dynamic energy absorption.

\section{INTRODUCTION}

Carbon nanotube composites are a topic of intense interest, motivated by the potential for enhancements of mechanical stiffness and strength, ${ }^{1,2}$ vibrational damping, ${ }^{3-5}$ and other properties, ${ }^{6,7}$ in polymer matrices. With respect to mechanical properties, attention has primarily been focused on quasistatic characterization, such as tensile, ${ }^{8-12}$ dynamical mechanical, ${ }^{5,12,13}$ or crack toughness ${ }^{13}$ measurements. Here, we focus on the impact response of polycarbonate (PC) nanocomposites.

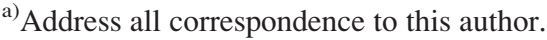

e-mail: Paul.Bruehwiler@empa.ch

${ }^{b)}$ This author was an editor of this journal during the review and decision stage. For the JMR policy on review and publication of manuscripts authored by editors, please refer to http://www.mrs. org/publications/JMR/policy.html
}

DOI: $10.1557 / J M R .2010 .0139$
The influence of carbon nanotubes (CNTs) on PC impact properties has not been reported. PC itself is well known for excellent impact energy absorption. Previous work has used Izod and Charpy tests, ${ }^{14-20}$ Taylor impact, ${ }^{21,22}$ plate and drop impact, ${ }^{23}$ split Hopkinson pressure bar (SHPB) in compression ${ }^{24,25}$ and tension, ${ }^{26}$ falling dart, ${ }^{27}$ ballistic, ${ }^{27}$ and projectile penetration ${ }^{28}$ measurements. The effects of nanofillers are less well studied. Particles of polyhedral oligomeric silsesquioxanes (POSS) were shown primarily to suppress the $\beta$-transition of polycarbonate in SHPB measurements, thereby decreasing the high strain rate dependence of the yield stress. ${ }^{25}$

Many aspects of a falling dart (penetration) test can be explained in terms of tensile response. ${ }^{29,30}$ This connection suggests that quasistatic tensile data should offer insight into falling dart results. Given the extensive studies of quasistatic mechanical properties, it is important to link these to impact studies. 
We compared the quasistatic tensile and impact mechanical properties of multiwall CNT (MWNT) composites of PC. We examined the effects of extrusion and of MWNT concentration near the electric and rheological percolation threshold of approximately $1-2 \% .^{13,31}$ Tensile tests were used to characterize the quasistatic, and falling dart tests the impact response. For the latter, we used the concept of an impact modulus to compare elastic properties.

We used optical microscopy and small-angle x-ray scattering (SAXS) to characterize the MWNT morphology and dispersion as a function of concentration. The linking of these methods allows us to characterize morphology over length scales from $1 \mathrm{~nm}$ to $1 \mathrm{~mm}$. Of particular interest is a new scanning SAXS analysis that is used to investigate the effects of extrusion on the dispersion of MWNT as a function of position in the extrudate.

\section{EXPERIMENTAL}

\section{A. Composite preparation}

The composite materials were prepared by melt mixing using a Micro 27 twin-screw extruder (Leistritz) with a length-to-diameter (L/D) ratio of 36 . A masterbatch containing $15 \mathrm{wt} \%$ MWNTs in polycarbonate, as provided by Hyperion Catalysis International (Cambridge, MA), was diluted using polycarbonate Lexan EXL6013 88225 (General Electric, now Sabic Innovative Plastics Europe), which is commonly used in high-impact applications, and contains a titania whitening agent. The nanotubes in this masterbatch are produced by chemical vapor deposition (CVD) and are characterized by diameters between 10 and $15 \mathrm{~nm}$ and pristine lengths between 1 and $10 \mu \mathrm{m}$. They were not purified before mechanical mixing in the masterbatch, so that small quantities of support and catalyst material from the CVD process were present in the masterbatch. Compounding was done using a screw speed of $200 \mathrm{rpm}$ and a throughput of $10 \mathrm{~kg} / \mathrm{h}$ using a standard screw for polycarbonate. The temperature profile ranged between $290{ }^{\circ} \mathrm{C}$ near the hopper to $270{ }^{\circ} \mathrm{C}$ at the die exit. The materials were added as granular premixtures after drying at $120{ }^{\circ} \mathrm{C}$ for at least $4 \mathrm{~h}$ in vacuum. The strands were extruded in a water bath and pelletized. Samples with final MWNT concentrations of 1.5\%, 2.0\%, and 2.5\% were produced; for comparison, a sample of pure polycarbonate (designated $0.0 \%$ ) was also extruded. The $0.0 \%$ samples were white and samples with MWNT black in color, all being opaque.

\section{B. Injection molding}

Injection molding was done using an Ergotech 100/ 420-310 injection molding machine (Demag), for which the melt temperature was $280{ }^{\circ} \mathrm{C}$, the tool temperature was set to $100{ }^{\circ} \mathrm{C}$, and a medium injection velocity was selected. The sample shapes were plates with dimensions of $80 \times 80 \times 3 \mathrm{~mm}^{3}$ and $80 \times 80 \times 4 \mathrm{~mm}^{3}$ and were injected by slits in pairs. An additional sample without MWNT was molded without prior extrusion, and is designated "NE" (not extruded).

\section{Optical micrographs}

Sections with a thickness of $5 \mu \mathrm{m}$ were cut from the center of a 3-mm-thick plate of each composition using a Leica 2055 microtome (Leica Mikrosysteme Vertrieb $\mathrm{GmbH}$, Bensheim, Germany) at room temperature and were fixed with Aquatex on glass slides. The optical microscopy investigations were performed using a $\mathrm{BH} 2$ microscope and a DP71 camera (Olympus Deutschland $\mathrm{GmbH}$, Hamburg, Germany) at various magnifications to detect agglomerates.

\section{Small-angle x-ray scattering}

SAXS measurements in transmission were carried out at beamline X12SA of the Swiss Light Source, ${ }^{32}$ with 3-mm-thick plates as samples. Each sample was measured at $80 \times 76$ matrix of points $1 \mathrm{~mm}$ apart, along and perpendicular to the injection direction, respectively. The sample was scanned continuously, at a wavelength of $1.000 \AA$, an exposure time of $0.1 \mathrm{~s}$ per point collected during the sample motion, and a constant $\mathrm{x}$-ray flux of approximately $10^{12} \mathrm{~s}^{-1}$ focused onto a spot of approximately $24 \times 8 \mu^{2}$. A pilot study had shown that exposures of $1 / 2$ and 5 times the present levels produced no detectable damage of the samples in SAXS or visually at the sample surface, which was also found in the current measurements; the agreement between those and the present results is excellent. The variations in scattering profile from position to position on the sample were minor. The images of the 6080 resulting x-ray patterns were each collected on a two-dimensional (2D) detector and directly stored for analysis. The images were then corrected for missing pixels by symmetry, and integrated azimuthally to yield the scattering intensity as a function of scattering vector and position on the sample. All analysis was carried out with MATLAB 7.3.0 for Windows.

Ultra-small-angle $\mathrm{X}$-ray scattering (USAXS) was carried out at the 32-ID-B beam line of the Advanced Photon Source (APS), Argonne National Laboratory, Argonne, IL. The USAXS instrument covers a much larger range of scattering angles and also measures the intensity on an absolute scale. The double-crystal instrument produces slit-smeared data, which were desmeared using APS routines. The x-ray wavelength was $1.00 \AA$. An air blank was subtracted from all the USAXS scattering patterns. 


\section{E. Tensile testing}

Quasistatic tensile tests were performed according to EN ISO 527-2 on a universal testing machine (Zwick Z010, Ulm, Germany). A 10-kN load cell and a mechanical extensometer with gauge a length of $25 \mathrm{~mm}$ were used. The measurements were performed at a constant crosshead speed of $2 \mathrm{~mm} / \mathrm{min}$ under standard climate $\left[23{ }^{\circ} \mathrm{C} / 50 \%\right.$ relative humidity $\left.(\mathrm{RH})\right]$ after conditioning for at least $24 \mathrm{~h}$. The nominal specimen dimensions of type 1 BA were $55 \mathrm{~mm}$ free length and $4 \times 5.5 \mathrm{~mm}^{2}$ cross section. To study the effects of tension parallel and perpendicular to the flow direction, five specimens were machined out of each of two 4-mm-thick injected plates for a given material preparation; they were $14.2 \mathrm{~mm}$ wide at the ends and $80 \mathrm{~mm}$ in total length, and were evenly distributed across the plates, separated by $2 \mathrm{~mm}$. The samples were numbered as follows: (i) in the parallel direction, samples 1 and 5 were taken from the sample edges and sample 3 from the middle, with 2 and 4 between; (ii) in the perpendicular direction, sample 1 was closest to the injection slit and 5 furthest away. The yield stress $\sigma_{\max }$ is calculated at the first maximum in the data, and the energy absorbed, or toughness, is the integral of the force-strain curve.

\section{F. Falling dart measurements}

Instrumented punch measurements were carried out using falling dart equipment (FractoVis Driven Mass falling dart: Ceast, Italy). The tests were performed according to ISO 6603 Part 2, with a dart total weight of $8 \mathrm{~kg}$, tip diameter $20 \mathrm{~mm}$, and support diameter $40 \mathrm{~mm}$. The specimens were unclamped. Oil was not used on the dart tip. The initial impact speed was $12 \mathrm{~m} / \mathrm{s}$, corresponding to kinetic energies in the range of $580 \mathrm{~J}$. The nominal specimen dimensions were $80 \times 80 \times 3 \mathrm{~mm}^{3}$. The load and displacement were recorded as a function of time and converted into load-displacement and energy-displacement curves. Three specimens per composite type were investigated.

To analyze the falling dart results in terms of sample stiffness, we considered the nature of the deformation process and the resulting curves. Nimmer ${ }^{29,30}$ showed that the deformation behavior exhibits analogies to a quasistatic tensile test, with primary qualitative differences attributable to the axisymmetric geometry and the deformation across the dart head, and quantitative differences due to rate-dependent yield stresses. Figure 4 of Ref. 33 illustrates a typical measurement with high-speed photography linked to a force-time curve, and Fig. 5 of Ref. 29 sketches the stretch states and distortions. Two stages of deformation are relevant for the present goals. (i) The dart, or striker, head initially deforms the portion of the sample in the immediate vicinity of the contact, inducing a $2 \mathrm{D}$ thinning and spreading of the material. (ii) When the portion of the sample in contact with the striker head strain hardens, an annular area around it is distorted. These stages are reflected in the observed material shapes after the measurement. ${ }^{30,33}$ The response of the material is complex due to the continuously changing geometry of the contact and surrounding sample, including relative motion. Nevertheless, the initial stress-strain relationship is linear, ${ }^{29,30,33}$ and a second linear portion can often be observed. ${ }^{29,30,33}$ In keeping with this observation, one may assume that much of the response is elastic, as found in simulations of ABS. ${ }^{34}$ The second linear region of the force-deformation curve can also be assumed to be largely elastic. ${ }^{29}$ We chose therefore to analyze the slopes of both portions of the force-deformation data as measures of the elastic modulus in the falling dart measurements.

\section{RESULTS AND DISCUSSION}

\section{A. Optical micrographs}

Because the present base polymer was of a different grade than in previous studies ${ }^{31,35}$ and contained a whitening agent, it was necessary to check the quality of nanotube dispersion and distribution. Optical microscopy investigations (see Fig. 1) revealed for the $0.0 \%$ sample that the whitening agent does not lead to agglomeration on that scale. Despite a seemingly good general dispersion and distribution of the nanotubes, however, some remaining agglomerates are visible at all MWNT concentrations. These agglomerates increase in number and size with concentration and can be up to $60 \mu \mathrm{m}$ in diameter. On the basis of previous experience with such masterbatches ${ }^{35}$ we assume these objects to be incompletely dispersed masterbatch agglomerates. Thus, we expect the nanotube concentration within the dark areas to vary between $15 \mathrm{wt} \%$ (masterbatch concentration) and the desired concentration. Obviously, the extrusion conditions used for the present masterbatch dilution, which were similar to those in Ref. 35, did not lead to perfect agglomerate dissolution, an observation that is confirmed by SAXS.

\section{B. Small-angle x-ray scattering}

Figure 2 displays the SAXS data summed over all points on the sample. SAXS is sensitive to charge density variations of the order of $2 \pi / q$. The scattering is not affected by extrusion, since both curves for pure PC $(0.0 \%$ and NE) overlap almost perfectly. This level of reproducibility is also found when comparing different plates at the same concentration, suggesting a high level of control in the sample preparation. We found that the masterbatch-induced scattering, deduced by subtracting the signal for the pure sample, is proportional to masterbatch concentration. This observation implies 


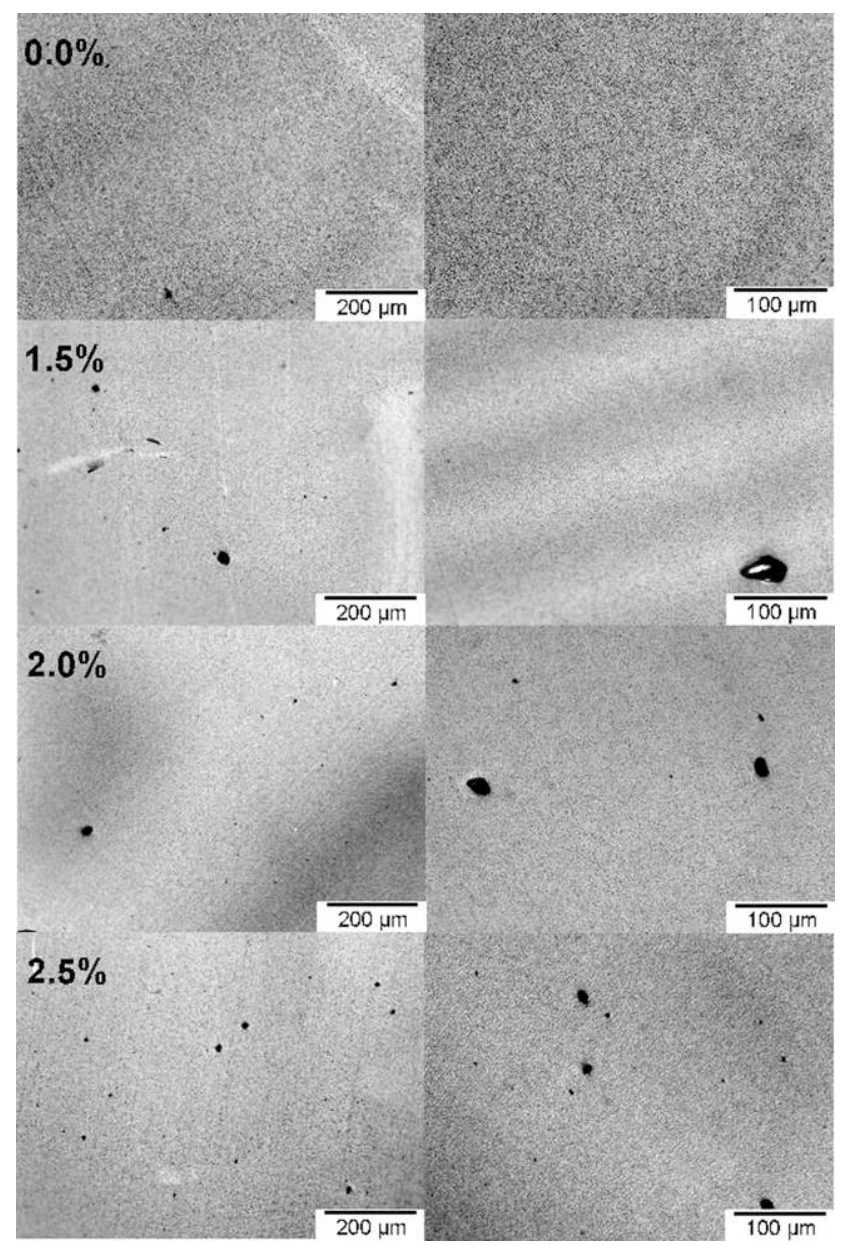

FIG. 1. Optical micrographs of 5- $\mu$ m-thick slices taken from the center of 3-mm-thick plates of the indicated compositions (note the different scales left and right).

that there are no qualitative differences in the MWNT dispersion or in the MWNT-induced matrix structuring among the concentrations studied at the scattering length scales.

The USAXS data shown in Fig. 3 confirm that scattering below $0.01 \AA^{-1}$ is dominated by the polycarbonate matrix and are consistent in the range of overlap with Fig. 2. In addition, all of these data show a Guinier rollover around $0.001 \AA^{-1}$. The low- $q$ region of the matrix material (0\%, EXL6013) was analyzed first. The data were fit to a unified function ${ }^{36}$ to extract the radiusof-gyration and the power-law slope at high $q$. The radius of gyration is $1960 \AA$, corresponding to a hard-sphere radius of $0.25 \mu \mathrm{m}$, a reasonable size for titania whitening agents.

Porod analysis ${ }^{37}$ was also carried out on the USAXS data for the matrix material to extract the titania particle interfacial area from the Porod constant, $B$, measured in the power-law region where the intensity $(I)$ follows Porod's law $\left(I \sim B / q^{4}\right)$ and the invariant $\left(Q_{\text {inv }}\right)$, which is

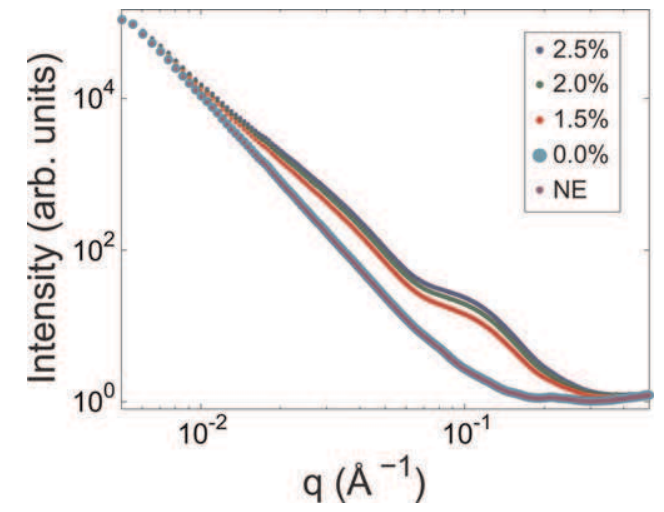

FIG. 2. SAXS data for the indicated samples. Note that the sample at $0.0 \%$ without extrusion (NE) strongly overlaps that of the extruded sample at $0.0 \%$, so that the latter is plotted with larger markers. (color online)

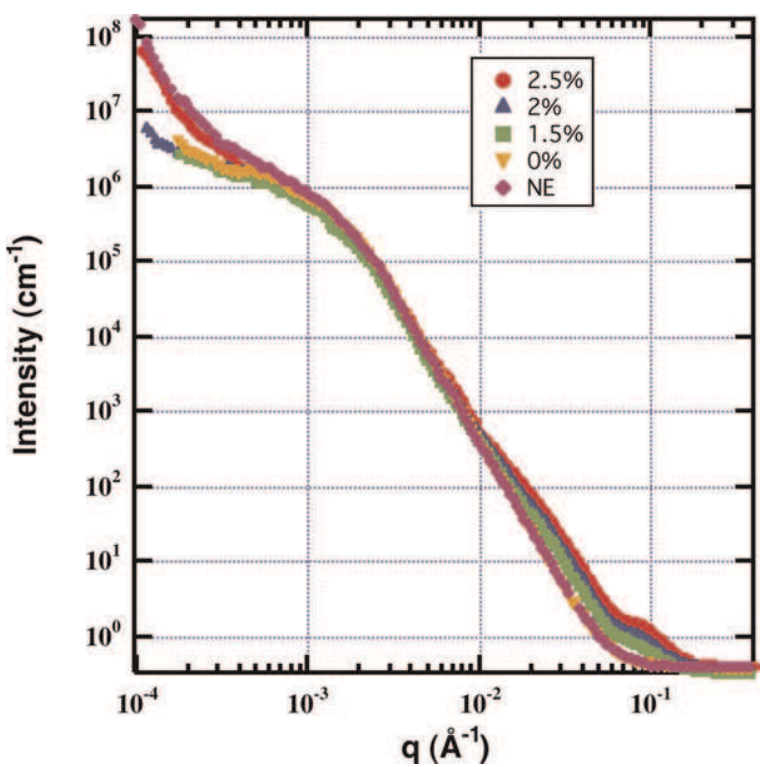

FIG. 3. USAXS data for the same samples as in Fig. 2. The upturn at small $q$ is probably due to surface irregularities. This region is ignored in the analysis. (color online)

obtained from an integral over $q^{2} I(q)$. The derived interfacial area per unit volume and equivalent hard-sphere radius of the particles are then $S_{\mathrm{v}}=\pi B \phi / Q_{\text {inv }}=0.11 \mathrm{~m}^{2} / \mathrm{cm}^{3}$ and $R_{\text {hard }}=Q_{\text {inv }} / \pi B=0.073 \mu \mathrm{m}$, respectively. The volume fraction, $\phi$, of the titania can be obtained from the invariant and the contrast in the scattering length density, $\Delta \rho$, which depends on the mass densities and compositions of the titania and matrix phases. Assuming a density of $4.22 \mathrm{~g} / \mathrm{cm}^{3}$ for titania and $1.18 \mathrm{~g} / \mathrm{cm}^{3}$ for the matrix $\left(\mathrm{C}_{16} \mathrm{H}_{14} \mathrm{O}_{3}\right), \phi=2 \pi^{2} Q_{\text {inv }} \Delta \rho^{2}=0.0027$. The particle radius derived from the Porod constant is smaller than that derived from the Guinier radius $\left(R_{\text {hard }}=0.253 \mu \mathrm{m}\right)$ because of the broad size distribution of the titania particles; the former is sensitive to the small particles and the latter is sensitive to the large particles. 
To study the effect of carbon, the $2.5 \%$ sample was analyzed in detail (Fig. 4) by subtracting the $0 \%$ data and fitting to various models for CNTs, ignoring the $18-\AA$ feature at high $q$. The low $q$ cutoff was chosen to exclude data for which the effects of surface and titania particle scattering were important. Since the limiting slope at low $q$ is not -1 , no rigid-rod or rigid-tube model can fit the data. Because the arrangement of the CNTs can be complex, we focused on deriving a general picture based on simple, established models. To account for flexibility and disorder in the MWNTs, we fit to fractal rod and fractal tube models. ${ }^{38,39}$ These models assume rodlike behavior for distances less than a persistence length and random fractal character for length scales larger than the persistence length. In both cases, however, the best fit is achieved with the persistence length comparable to the diameter of the rods in the model, which, within our assumptions, implies there is no rodlike character at all. The fractal rod model is shown in Fig. 4. The fact that we can fit the data with reasonable parameters implies that the scattering does arise from carbon. The nanotubes, however, are highly defective and highly disordered. ${ }^{40}$ This behavior is often observed in systems where harsh strategies are used to disperse the nanotubes. The remaining feature at high $q$ is associated with the masterbatch, but is probably not due to carbon. This $18-\AA$ feature could be due to residual catalyst particles or some other additive included by the masterbatch manufacturer.

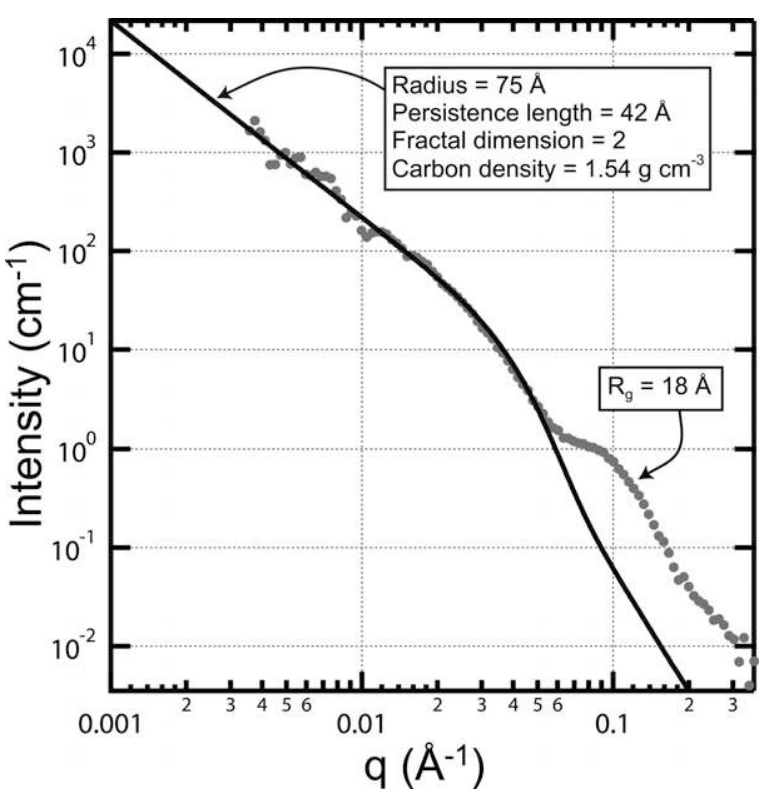

FIG. 4. The background-subtracted 2.5\% data of Fig. 3 fit to a fractal rod model. The high- $q$ feature was ignored in the fit. Since the persistence length is comparable to the rod diameter, no rodlike character is present. The fractal dimension of 2.0 implies that the tubes are highly disordered on lengths larger than the diameter. The matrix was assumed to be $\mathrm{C}_{16} \mathrm{H}_{14} \mathrm{O}_{3}$ with a density of $1.18 \mathrm{~g} / \mathrm{cm}^{3}$. A carbon density of $1.54 \mathrm{~g} / \mathrm{cm}^{3}$ was assumed. The fit is weakly dependent on the details of the assumed composition.
Figure 5 displays the SAXS intensity as a function of position on the sample collected at 1-mm intervals, as described in Sec. II. D; the data were averaged over the $q$-ranges shown. Since the results for the NE were similar in all details to those of the $0.0 \%$ material, the former are not shown. The polymer injection occurred from the right in the perspective of the figure, which is apparent in the data as a lower scattering intensity, consistent with lower material density variations at the inflow point. These patterns were highly reproducible for a given material, with minor differences between members of a given pair. The apparent contours roughly reflect the filling front during injection molding, which was previously studied for polycarbonate-MWNT composites. ${ }^{41}$

The lowest $q$-range is shown in Fig. 5(a), corresponding to the largest length scale of material density variations accessible in the scanning measurements, of the order of $0.1 \mu \mathrm{m}$. These data are qualitatively similar for all the materials, and confirm a high level of reproducibility in the sample production. The data for the $0.0 \%$ material cover a larger intensity range. Since there are almost negligible masterbatch-induced scattering
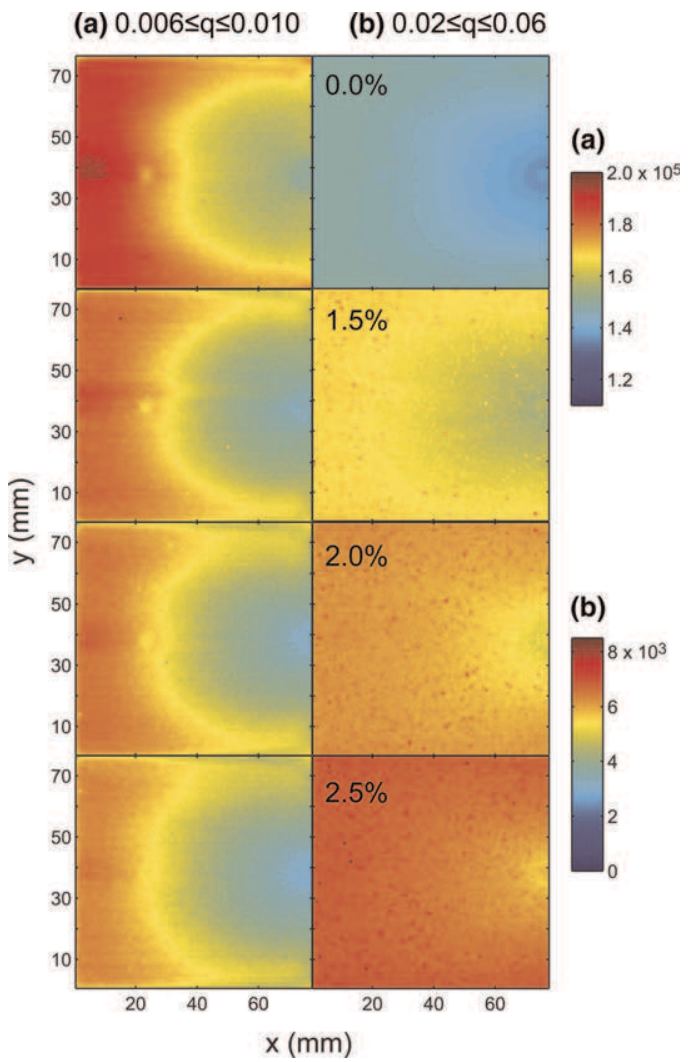

FIG. 5. Position-resolved average SAXS intensity of the indicated samples. The data in each column $(\mathrm{a}, \mathrm{b})$ represent the entire sample divided into 1-mm-square regions, in each of which a SAXS scan was acquired and averaged over the $q$-range indicated. Melt injection occurred from the right. The color scale for each column is given in counts. (color online) 
contributions in this $q$-range, the matrix polymer is similarly distributed for all the samples.

As discussed in connection with Fig. 4, Fig. 5(b) corresponds to the $q$-range of the broad MWNT-induced shoulder in the SAXS data. Immediately apparent is the large increase in scattering in this range, which varies monotonically with the concentration. The $2.0 \%$ and $2.5 \%$ materials yield qualitatively similar data, with the $1.5 \%$ sample exhibiting an intensity distribution with elements in common with the $0.0 \%$ sample. All three MWNT-containing samples show evidence of noticeably higher scattering at particular spots, with more of these for the $2.0 \%$ and $2.5 \%$ materials. This feature is a clear footprint of an inhomogeneous distribution of the MWNTs, i.e., agglomeration on a length scale smaller than the collection interval of $1 \mathrm{~mm}$, but at least of the order of the x-ray beam diameter of about $10 \mu \mathrm{m}$, in accordance with the more local analysis of Fig. 1. Thus, site-dependent SAXS yields a global assessment of the presence of agglomerates, which are found throughout all MWNT-containing samples.

\section{Tensile tests}

Figure 6 shows the raw tensile data, differentiating between samples taken parallel and perpendicular to the injection direction. The sample numbering increases away from the injection point for those taken perpendicular, and from one (arbitrary) side to the other for those taken parallel. Polycarbonate under quasistatic strain typically exhibits yielding behavior with necking, and strain hardening at larger strains before breaking. ${ }^{26,42}$ This behavior is observed for many of the samples. Small variations are apparent in both the initial linear portion and the yield stress, and larger variations in terms of strain-atbreak and strain hardening. Overall, the variation from position to position for a given MWNT concentration and tensile orientation varies more strongly at higher concentrations, and strain-to-break for the samples taken parallel to the injection direction is lower. Since the plateaus are similar, these data already give an impression of the ductility, or energy absorption. Samples containing $2.5 \%$ MWNT are the most brittle in the present series, whereas the difference between $1.5 \%$ and $2.0 \%$ is smaller.

One effect of sample extraction direction is the difference in the initial yielding behavior for the NE and $0.0 \%$ samples. In the parallel direction, the decrease from the initial maximum to the plateau occurs more rapidly, being complete on average at a strain of 0.07 , whereas this decrease occurs at strains well over 0.10 in the perpendicular direction. Similar effects have been exhibited by polyethylene and polypropylene. ${ }^{43}$

The variation in strain-at-break for these samples is not easy to explain. The starting polymer (EXL6013) contains titanium oxide particles. Similar oxide particles have been shown to affect the mechanical properties of polycarbonate, ${ }^{14}$ but not greatly at low concentrations. This behavior is confirmed here in that the yield stress is in all cases similar to that found in other studies of pure polycarbonate (see, e.g., Refs. 26 and 44) and titania-free grades of this polymer (see manufacturer data for Lexan

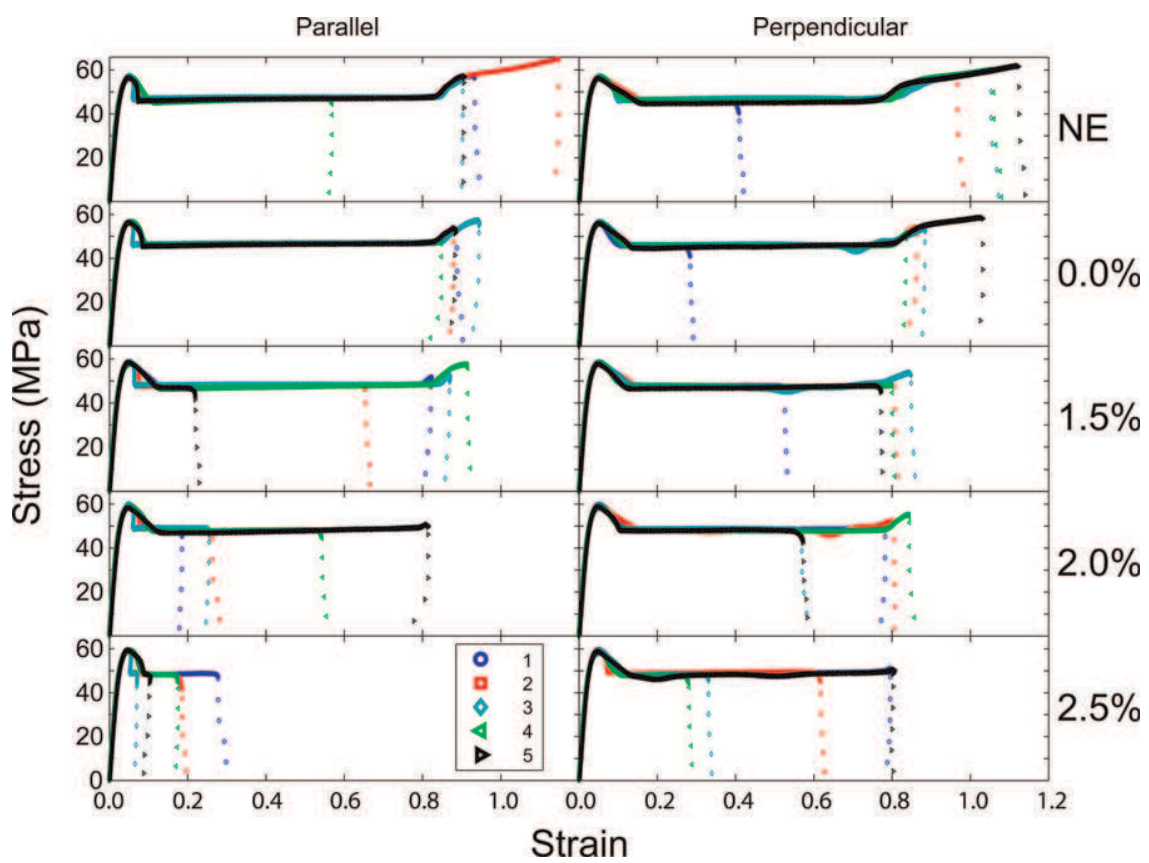

FIG. 6. Quasistatic tensile data of all samples, with the direction of the tension with respect to the injection direction shown. The position (1-5) of the sample in the original plate, as described in Sec. II. E, is also indicated. (color online) 
EXL 1413T or 1810T). Furthermore, the largest values of strain-at-break found here for masterbatch-free samples are similar to the titania-free grades, which exhibit values of 1.2 to 1.3 . This similarity indicates that the whitening agent in the matrix polymer had at most a minor influence on the mechanical properties. Hence, effects of the injection-molding process must be considered. Strong effects on the nm-scale structure, distributed throughout the sample, are apparent in Fig. 5. A role for processing as a source of tensile property reduction is suggested in the consistently low performance of position 1 in the perpendicular direction (for samples NE, $0.0 \%$ and $1.5 \%$ ), which is also where the largest scattering intensity variations are observed. We discuss this issue below.

Figure 7 summarizes the engineering yield stress and Young's modulus results. Both properties increase with MWNT concentration. The injection direction plays a ples and increasing the variability of the yield stress, though not significantly affecting the average value for a given concentration. No average effect of extrusion is evident in the modulus, though the variability increases; the average increases almost linearly with concentration, with saturation for the perpendicularly cut samples at $2.5 \%$. The yield stress data also show a saturation effect near $2.0 \%$ for both sample directions, and a significant difference between NE and $0.0 \%$ in the parallel direction. slight role as well, affecting the stiffness for some sam-

The modulus data for the parallel samples in Fig. 7 follow $E(\mathrm{GPa})=2.28(1+0.0377 \times \mathrm{wt} \%)$ as shown; expressed in terms of volume fraction, $\phi$, with an assumed MWNT density of $1.5 \mathrm{~g} / \mathrm{cm}^{3}, E(\mathrm{GPa})=2.28(1+2.51 \phi)$. The linear trend can be analyzed using a flexible-rod model [Eq. (15) of Ref. 39], which assumes that defects limit the effective length of the MWNT to shorter sections. In general, defects, which are intrinsic or due to processing, lower the potential for MWNT to reinforce the polymer matrix. This reduced reinforcement potential has so far not been apparent, e.g., in essential work of fracture studies on polycarbonate using the same masterbatch. ${ }^{13}$ In addition, the matrix-MWNT adhesion is good for cases of MWNT/polycarbonate composites reported so far, ${ }^{31,45}$ including the present masterbatch, so that poor MWNTmatrix adhesion does not seem to be important in the present case. Thus, the present model appears to be a reasonable approach to assess the role of defects. It suggests that the observed loading dependence corresponds to an aspect ratio of 6.3 , much lower than the native aspect ratio of the MWNT. This result is consistent with the USAXS data analyzed previously. Thus, we suggest that, because of disorder induced by processing, the individual MWNT are not effective modulus enhancers in the present samples. ${ }^{39}$ This observation provides the motivation to examine the energy absorption, which could conceivably be less compromised by disorder.

Figure 8 displays the energy absorption dependence on MWNT concentration. It is apparent that energy
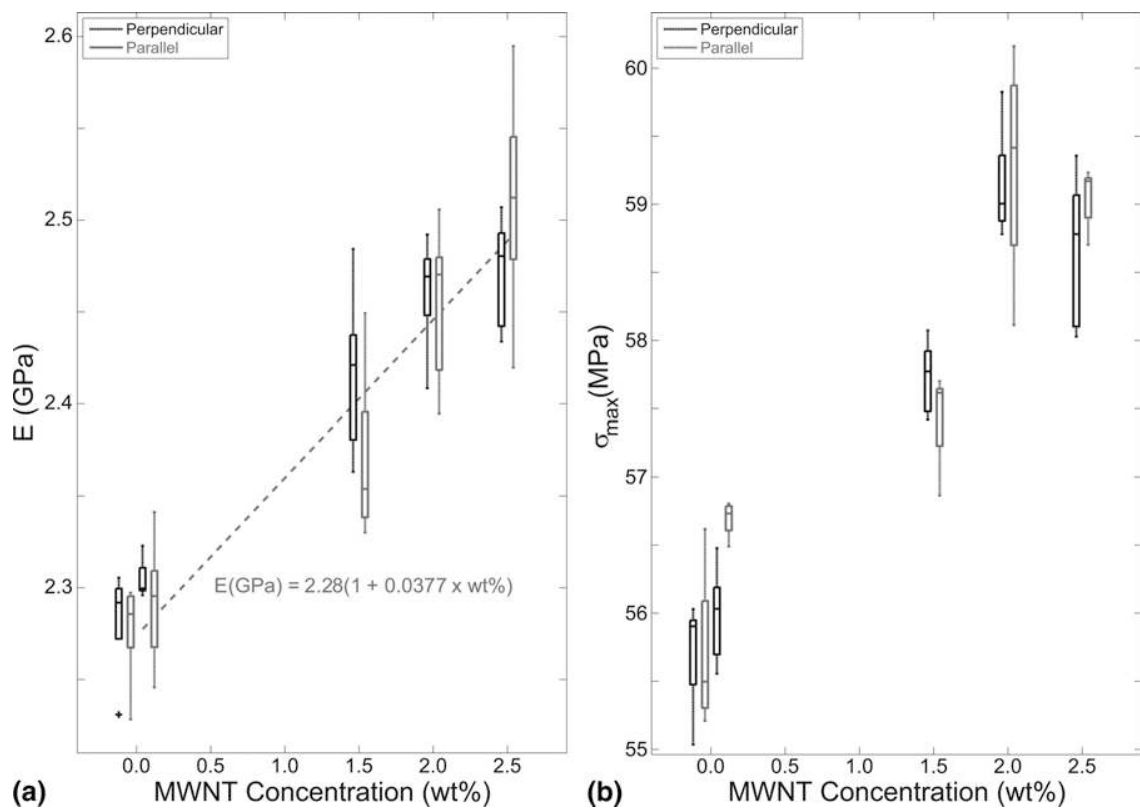

FIG. 7. Boxplots of the tensile (a) modulus and (b) yield stress as a function of MWNT concentration, including all samples. The sample orientation with respect to the injection direction is indicated. The NE material is represented by the points at the concentration $0.0 \%$ furthest to the left, and the $0.0 \%$ material to their right. The line and corresponding equation in (a) are a fit to the data for the parallel samples and are discussed in the text. (A boxplot is often used when there are few observations and it is unclear if the data are normally distributed. The central horizontal line in each box is the median, the upper and lower box edges represent the first upper and lower quartiles, respectively, and the whiskers ending in horizontal bars mark the second upper and lower quartiles. Outliers are indicated with "+" symbols.) 


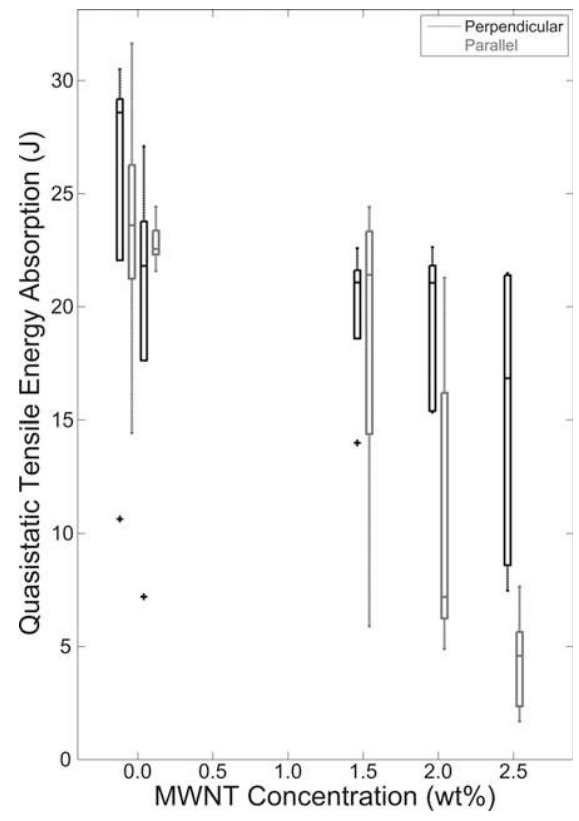

FIG. 8. Boxplot of the tensile energy absorbed as a function of MWNT concentration, including all samples. The direction of tension with respect to the injection direction is indicated. The NE material is represented by the points at the concentration $0.0 \%$ furthest to the left, and the $0.0 \%$ material to their right. See the caption of Fig. 7 for a description of boxplots.

absorption decreases with loading. The lowering of the energy absorption is mainly caused by the reduction in elongation at break, since the modulus and strength are constant or slightly increased with CNT addition. Increasing MWNT concentration is associated on average with lower energy absorption, as also observed for nanosilicate-PC nanocomposites. ${ }^{46}$ The sample orientation plays a visible role in this regard as well, when the MWNT concentration reaches $2.0 \%$ or above. A more localized shift with MWNT concentration is suggested in the individual values as a function of position.

For the perpendicularly extracted samples, sample 1 (closest to the injection-molding gate) exhibits values less than half of the other samples for materials NE and $0.0 \%$, whereas at $1.5 \%$ sample 1 displays a value approaching that of the others. For higher concentrations, sample 1 achieves one of the highest values for the material. Sample 5 (farthest from the gate) generally exhibits one of the highest values at all concentrations. The good reproducibility of these observations for the two samples $\mathrm{NE}$ and $0.0 \%$ suggest that the pure samples may be compared to other work. Notably, the gross differences in energy absorption exhibited by the positions closest to and farthest from the gate were also observed for injection-molded polyethylene. ${ }^{43}$ Although polyethylene is semicrystalline and polycarbonate amorphous, one can assume that injection molding of such thermoplastic polymers could induce analogous variations in the global structure and alignment of the material.
For parallel extraction sample 2 exhibits the highest absorbed energy for material NE, and moves steadily toward lower values with MWNT concentration. Similarly, samples 1 and 4 consistently show among the highest values for materials NE, $0.0 \%$ and $1.5 \%$, and then dropping strongly for materials $2.0 \%$ and $2.5 \%$. The consistency of these observations suggests that sample-to-sample variability is not a dominant factor. An apparent deviation from the polyethylene results is observed when comparing perpendicular to parallel, for which a sample position equivalent to the present sample 3 showed notably higher energy absorption than all perpendicular samples. ${ }^{43}$ Similar results were found for polypropylene samples in nonessential work of fracture measurements, ${ }^{47}$ giving consistently higher values for samples obtained parallel to the injection direction, with or without MWNT. Hence, the effect of sample extraction with respect to the injection-molding direction on the mechanical properties is qualitatively different in this work compared to previous work on semicrystalline polymers, but qualitatively similar with respect to position in the plates for the perpendicular case. Note that the electrical conductivity of such samples may vary strongly throughout the plate as well, ${ }^{35}$ which could be attributed to local effects of agglomeration and/or polymer flow during injection.

Here, we attempt to correlate the observed differences among the samples containing different MWNT concentrations. In Fig. 5(b), a region of lower intensity scattering in the injection region is obvious for the $0.0 \%$ sample, less for the $1.5 \%$ sample, and essentially absent for the $2.0 \%$ and $2.5 \%$ samples. In Fig. 7(b), the approximately linear increase of yield strength with MWNT concentration ceases at $2.0 \%$ for samples of both orientations. Finally, Fig. 8 shows that the median energy absorption for parallel orientation diverges strongly from the perpendicular median value at $2.0 \%$. This concentration range is that identified as encompassing the percolation threshold for similar samples using different matrix PC grades $^{13,31}$ and has been linked to a tough-to-brittle transition above $2 \%$ in a study of crack strength using the same masterbatch material. ${ }^{13}$ As already noted, the slight inhomogeneities evident in Fig. 5(b) are stronger and qualitatively similar for concentrations above $1.5 \%$. Hence, in addition to MWNT network formation, the density of agglomerates in our samples must be considered as an explanation for the observed trends in the mechanical behavior. These alternatives have both been discussed previously to explain a similar evolution of the mechanical properties with nanoparticle concentration. ${ }^{14,46,48,49}$ It is striking that other studies of PC reinforcement also showed maxima in selected mechanical properties at concentrations of a few percent of carbon nanotubes ${ }^{12,50-52}$ or nanofibers. ${ }^{8}$ In most of these studies, difficulties in achieving uniform dispersion of the 
nanoparticles at higher concentrations were identified as the cause of the associated mechanical degradation. Hence, we must conclude that both nanotube networking and aggregation may contribute to the presently observed changes around a concentration of $2 \%$.

Figure 9 shows that the MWNT-containing samples exhibit much more structured tensile fracture surfaces. These surfaces are generally characterized by a number of small holes and/or funnel-shaped deformations originating at a structure less than a few tenths of a millimeter in diameter. We have not attempted to investigate these defects in detail, but attribute them to the presence of the masterbatch-induced defects deduced from optical microscopy and SAXS. There is, however, no clear increase in defect density with masterbatch concentration.

Considering other work on energy absorption in nanocomposites, polyethylene modified by clay showed significant decreases at a filler concentration of $5 \%,{ }^{53}$ with no information on intermediate concentrations. Polyamide-12 exhibited decreases in modulus and strength, as well as stress- and strain-at-break for layered silicate concentrations above $1 \%$, which was attributed to the development of a percolation network. ${ }^{54}$ These works support that general assertion ${ }^{55}$ that nanofillers in polymers decrease energy absorption in fracture.

\section{Falling dart}

Figure 10 shows the results of the falling dart measurements. The reproducibility is high except near the maximum force $\left(F_{\max }\right)$. The oscillations in the curve are also well reproduced from measurement to measurement. $F_{\max }$ and energy absorption of the material decreased on incorporation of MWNT. At the same time, the penetra-

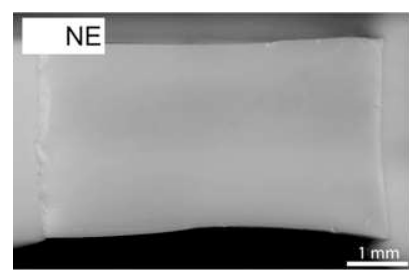

(a)

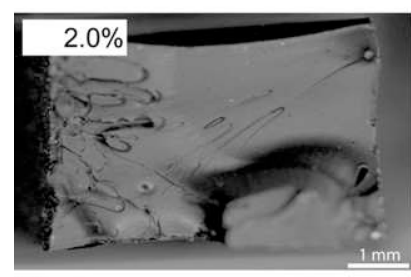

(c)

FIG. 9. Optical images $(20 \times)$ of fracture surfaces of sample 1 in the parallel orientation for the indicated materials, which are representative for all cases. The surfaces are of the order of $5 \times 3 \mathrm{~mm}^{2}$ in area. All MWNT-containing samples are in reality the same black color, and the NE sample is white; in optimizing the contrast, an apparent discoloration took place. tion behavior is modified, with longer distance needed after initial penetration until the striker is unloaded $(F=0)$. The absorbed energy values, calculated by integration of the exerted force up to the zero crossing, are shown in Fig. 11.

During the initial phase of the load-displacement curve, oscillations are observed due to the vibrations of the striker after contact. It is nevertheless possible to apply a linear fit to this part of the curve, as well as to a second portion above 5-mm displacement, as discussed

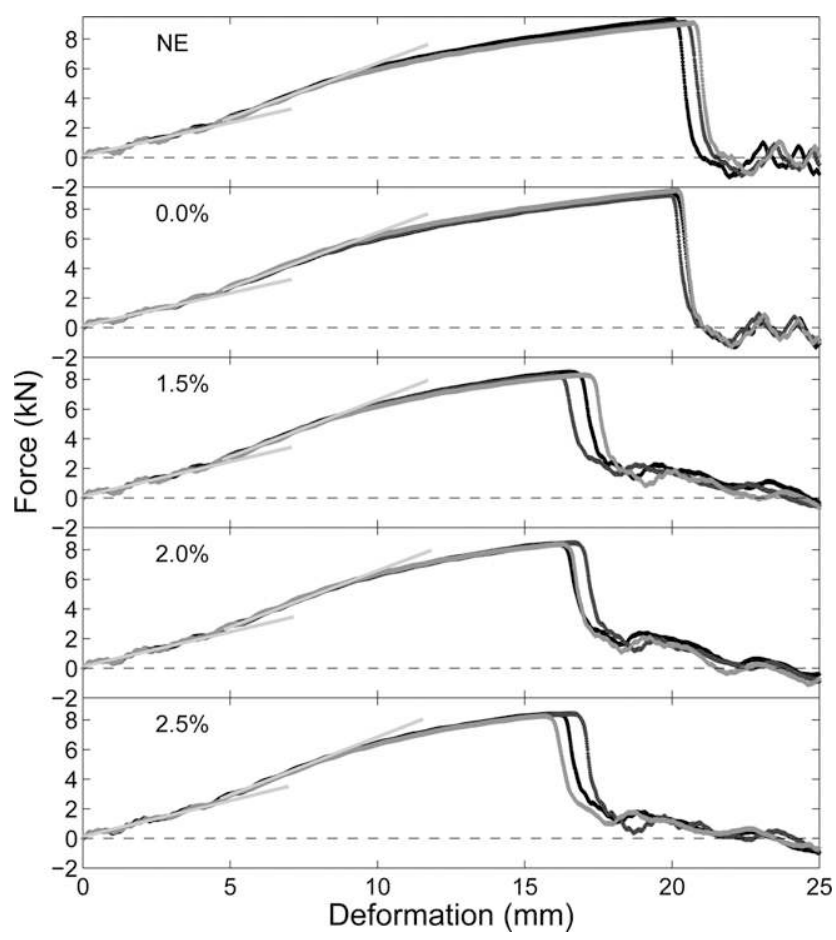

FIG. 10. Summary of the falling dart measurements. The lines indicate the results of fitting straight lines to the initial increase of the force and a secondary linear hardening, as described in the text. The fits are projected to higher deformations to better visualize their ranges of validity.

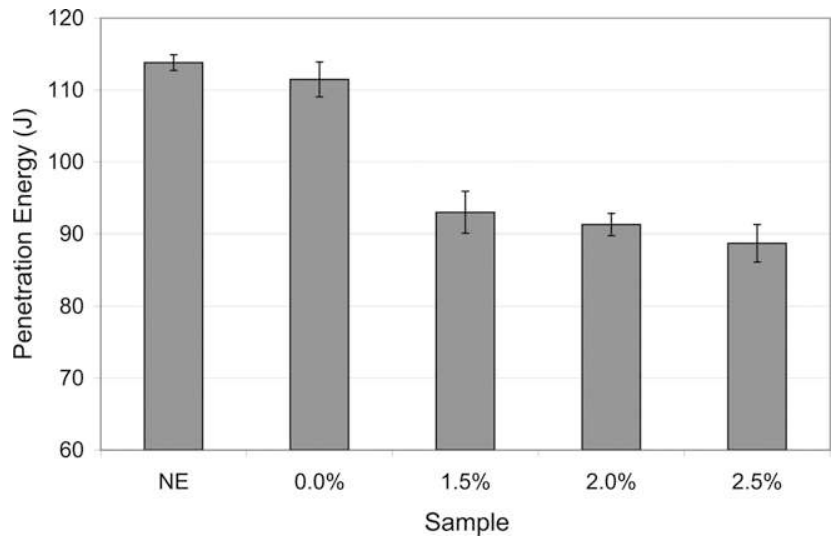

FIG. 11. Penetration energy for the indicated samples with standard deviations. 
in Sec. II. F. The green lines in Fig. 10 show the results of this analysis. The data were fitted over the ranges $0-3.5$ and $4.7-8.2 \mathrm{~mm}$; the fits are shown over greater ranges to illustrate where the data deviate from linearity. The slopes cannot be converted to true elastic moduli without a more detailed understanding of the polymer deformation, therefore in what follows we consider only their relative variations as a function of sample preparation.

Figure 12 summarizes all mechanical measurements reported here; the tensile data were averaged over both extraction directions. The effective impact moduli, designated $E_{1}$ and $E_{2}$ for the initial and secondary linear stress regions vary more or less in parallel, justifying our deduction previously that they measure similar quasielastic moduli. The fact that the impact moduli yield a trend comparable to the standard modulus determined in quasistatic tension indicates that changes in elastic properties, as measured here, are not a strong function of the deformation rate. The variation of the stiffness is approximately linear with the concentration, though with large fluctuations.

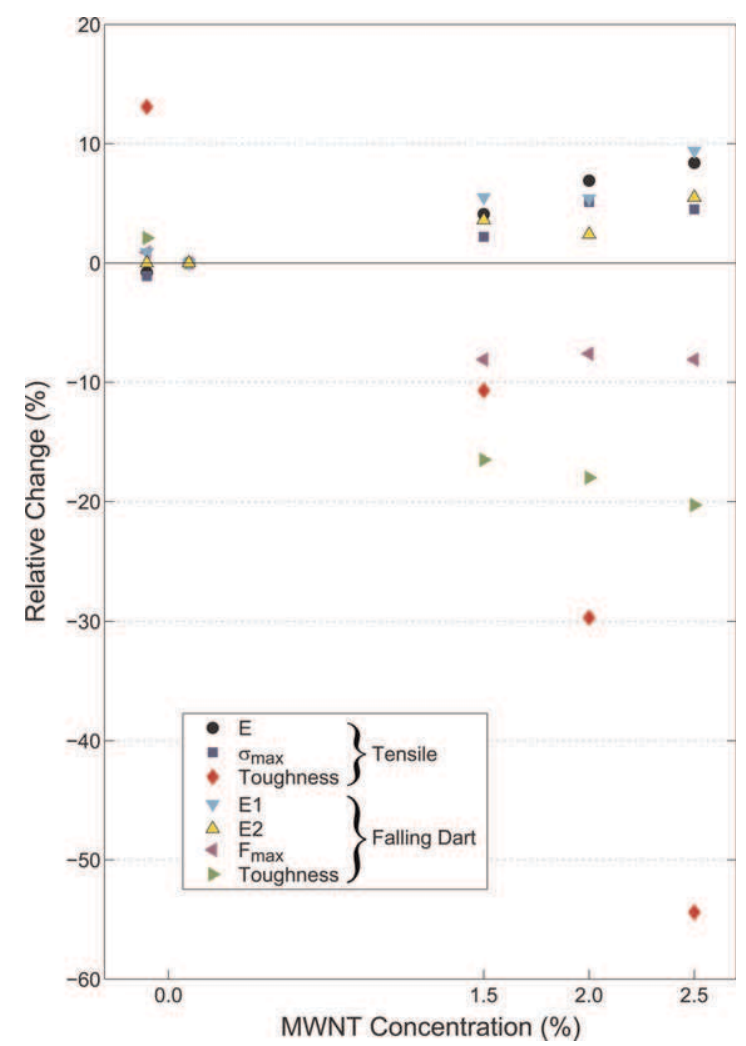

FIG. 12. Summary of the changes in mechanical properties relative to the extruded, $0.0 \%$ sample, for the indicated properties and samples. $E$ designates Young's modulus in quasistatic tension, $\sigma_{\max }$ the maximum stress in quasistatic tension, Toughness- $T$ the energy absorbed in quasistatic tension, $F_{\max }-\mathrm{FD}$ the maximum force in falling dart measurements, Toughness-FD the energy absorbed in the falling dart measurements, $E_{1}$ the initial elastic slope and $E_{2}$ the secondary elastic slope in the falling dart measurements. (color online)
The energy absorption data also show qualitatively similar effects, with a decrease on extrusion, and a much larger decrease as a function of MWNT concentration. In the quasistatic case, the effect of MWNT concentration appears to be strongly nonlinear, requiring an exponential function to achieve a curve passing through the origin. This nonlinearity is most strongly determined by the data from samples taken parallel to the injection direction. The falling dart energy absorption data are approximately a linear function of concentration, but with an intercept far below the observed value. This observation makes it clear that the methods study different aspects of the material behavior. A phenomenological rationalization is the complex 2D stretching of the material in the falling dart test. ${ }^{29,30}$ Not only does this stretching mix all possible extraction directions in an undetermined fashion, but apparently reduces the sensitivity to material defects. Microscopic examination of the falling dart fracture surfaces does not show the same dramatic differences between pristine and filled polymer, though the surfaces are somewhat more structured in the latter case, consistent with Fig. 9. This smaller difference is also in line with the greater consistency among the stress-strain curves observed.

The force $F_{\max }$ decreases on addition of MWNT, whereas the tensile yield strength $\sigma_{\max }$ increases. We attribute this difference to the different mechanisms involved. $F_{\max }$ as given in the standard falling dart analysis corresponds to strength-at-break. To estimate falling-dart-test yield strengths, we analyzed the point of departure from linearity corresponding to the determinations of $E_{1}$ and of $E_{2}$. This point was found to occur closely at the same level of penetration in a given region (1 or 2), regardless of MWNT concentration. Taking this point as an approximate yield point and evaluating the force (from the data or using $E_{1}$ or $E_{2}$ ) shows a similar trend as for $\sigma_{\max }$.

In summary, similar trends emerge in both the quasistatic and the impact measurements-the elastic quantities (stiffness, yield strength) increase with MWNT concentration and are relatively insensitive to extrusion effects. Energy absorption decreases slightly with extrusion and strongly with MWNT concentration. Qualitatively, however, there are differences between the impact and quasistatic measurements. For energy absorption the quasistatic results are more sensitive to both material history and MWNT concentration. Nevertheless, the impact tests indicate greater brittleness on inclusion of MWNT. ${ }^{5,56}$ Agglomerates associated with the MWNTmasterbatch have been observed directly in this work over micro- and macroscopic regions of the samples, and appear to be origins for fracture processes in the quasistatic tensile tests.

To place the present results in context, Table I offers a selection of data from the literature, in which the 
TABLE I. Nanocomposite mechanical parameters from the literature with relevance for impact characterization for polymer composites and exhibiting characteristics similar to the present materials.

\begin{tabular}{|c|c|c|c|c|c|}
\hline & & \multicolumn{3}{|c|}{ Quasistatic tension } & \multirow{2}{*}{$\frac{\text { Impact }}{F_{\max }}$} \\
\hline \multicolumn{2}{|c|}{ Material } & E & $\sigma_{\max }$ & Toughness & \\
\hline This work & $(2 \mathrm{wt} \%)$ & $+7 \%$ & $+5 \%$ & $-30 \%$ & $-8 \%$ \\
\hline$n$-clay/ABS ${ }^{56}$ & $(4 \mathrm{wt} \%)$ & $0^{\mathrm{a}}$ & $+5 \%^{\mathrm{a}}$ & $-61 \%^{\mathrm{b}}$ & $-94 \%^{\mathrm{b}}$ \\
\hline$n-\mathrm{Al}_{2} \mathrm{O}_{3} / \mathrm{ABS}^{59}$ & $(1 \mathrm{wt} \%)$ & $+9 \%{ }^{\mathrm{c}}$ & $-7 \%^{\mathrm{c}}$ & $-22 \%^{\mathrm{c}}$ & $-27 \%^{\mathrm{d}}$ \\
\hline$n$-clay/PP ${ }^{58}$ & $(4 \mathrm{wt} \%)$ & $+3 \%^{\mathrm{e}}$ & $+1 \%^{\mathrm{e}}$ & & $-70 \%{ }^{\mathrm{f}}$ \\
\hline $\mathrm{MWNT} / \mathrm{PP}^{57}$ & $(2 \mathrm{wt} \%)$ & $+35 \%^{\mathrm{g}}$ & $26 \%^{\mathrm{g}}$ & $-70 \%^{\mathrm{g}}$ & $-30 \%^{\mathrm{h}}$ \\
\hline
\end{tabular}

Note: In cases where multiple concentrations were studied that corresponding to a maximum property value or representing the overall trend was chosen.

${ }^{\mathrm{a} E s t i m a t e d ~ f r o m ~ F i g . ~} 5$ of Ref. 56.

${ }^{b}$ Estimated from Fig. 3 of Ref. 56. Unnotched Charpy test. Similar changes were found with a notched Charpy test.

${ }^{c}$ Estimated (toughness) or directly from Table 4 of Ref. 59. Note a slight discrepancy with Fig. 12 there.

${ }^{\mathrm{d}}$ Notched Izod test.

${ }^{\mathrm{e}}$ From Fig. 3 of Ref. 58. Comparison of RR7 to neat PP-Co M710, a highimpact copolymer.

${ }^{f}$ From Fig. 5 of Ref. 58. Comparison of RR7 to neat PP-Co M710. Notched Charpy test.

${ }^{g}$ From Table 1 of Ref. 57. Energy estimated, based in part on Fig. 5.

${ }^{\text {h}}$ Estimated from Fig. 7 of Ref. 57 (unnotched Charpy test). Notched Charpy results show an increase.

effects of nanofillers on quasistatic tensile and on impact parameters are compared. ABS is a well-known impact-resistant copolymer. Polypropylene (PP) has been studied often and has shown results qualitatively similar to PC in tensile tests, motivating its presence in the table. Impact modulus and energy absorption were not available in any of these publications. ABS shows strong similarities to the present results, with small changes in quasistatic modulus and strength, but large decreases in quasistatic tensile energy absorption and impact strength. Nanoclay and MWNT in PP exhibit similar behavior. Given the differences in morphology and dispersion methods and the difficulties in obtaining a good dispersion in most of these cases, ${ }^{56-58}$ agglomerates must be assumed to be present in those works, as well. In any case, the present results are comparable to those of similar recent studies on nanoparticle-polymer composite mechanical properties.

\section{CONCLUSIONS}

Falling dart tests of MWNT-filled polycarbonate reveal an embrittlement of the composites with MWNT content. We attribute at least some of this mechanical degradation to the presence of remaining masterbatchinduced agglomerates, as seen in optical microscopy and position-resolved SAXS investigations. MWNT network percolation may also contribute to the observed brittleness. We have further shown that it is possible to extract information about the stiffness of the composite under impact conditions from a falling dart test. Quasistatic tensile tests correlate well with the impact data for elastic parameters, but are much more sensitive to sample preparation for energy absorption. Small-angle x-ray scattering has been shown to be useful in scanning mode to derive information on local filler concentrations on a global scale.

To elucidate the relative roles of aggregates and percolation in the observed trends, samples with nearperfect filler dispersion would be required, which are presently difficult to obtain by melt mixing on the necessary kilogram scale. The analytical developments illustrated here (falling dart modulus, global filler concentration fluctuations in SAXS) could be further exploited. For nanocomposites, the latter would appear to be a particularly powerful approach, especially if the $\mathrm{x}$-ray beam can be focused to length scales comparable to those of typical agglomerates, so that a combination of long-range and short-range scans could map the particle distributions in greater detail.

\section{ACKNOWLEDGMENTS}

We gratefully acknowledge B. Kretzschmar for help with the extrusion and injection molding experiments; M. Heber for the optical microscopy investigation; K. Ruf, M. Heusser, and L. De Boni for carrying out mechanical measurements; J. Ilavsky for his efforts on the USAXS experiments; and A.J. Brunner for helpful and stimulating discussions. The use of the Advanced Photon Source was supported by the U.S. Department of Energy, Office of Science, Office of Basic Energy Sciences, under Contract No. DE-AC02-06CH11357.

\section{REFERENCES}

1. P.M. Ajayan and J.M. Tour: Materials science: Nanotube composites. Nature 447, 1066 (2007).

2. R.H. Baughman, A.A. Zakhidov, and W.A. de Heer: Carbon nanotubes-The route toward applications. Science 297, 787 (2002).

3. M.L. Auad, M.A. Mosiewicki, C. Uzunpinar, and R.J.J. Williams: Single-wall carbon nanotubes/epoxy elastomers exhibiting high damping capacity in an extended temperature range. Compos. Sci. Technol. 69, 1088 (2009).

4. X. Zhou, E. Shin, K.W. Wang, and C.E. Bakis: Interfacial damping characteristics of carbon nanotube-based composites. Compos. Sci. Technol. 64, 2425 (2004).

5. J. Suhr and N. Koratkar: Energy dissipation in carbon nanotube composites: A review. J. Mater. Sci. 43, 4370 (2008).

6. W. Zhang, R.C. Picu, and N. Koratkar: The effect of carbon nanotube dimensions and dispersion on the fatigue behavior of epoxy nanocomposites. Nanotechnology 19, 285709 (2008).

7. J. Suhr, P. Victor, L. Ci, S. Sreekala, X. Zhang, O. Nalamasu, and P.M. Ajayan: Fatigue resistance of aligned carbon nanotube arrays under cyclic compression. Nat. Nanotechnol. 2, 417 (2007). 
8. Y. Gao, P. He, J. Lian, L.M. Wang, D. Qian, J. Zhao, W. Wang, M.J. Schulz, J. Zhang, X.P. Zhou, and D.L. Shi: Improving the mechanical properties of polycarbonate nanocomposites with plasma-modified carbon nanofibers. J. Macromol. Sci. Part B Phys. 45, 671 (2006).

9. C.K. Kum, Y.T. Sung, M.S. Han, W.N. Kim, H.S. Lee, S.J. Lee, and J. Joo: Effects of morphology on the electrical and mechanical properties of the polycarbonate/multi-walled carbon nanotube composites. Macromol. Res. 14, 456 (2006).

10. P. Pötschke, H. Brünig, A. Janke, D. Fischer, and D. Jehnichen: Orientation of multiwalled carbon nanotubes in composites with polycarbonate by melt spinning. Polymer (Guildf.) 46, 10355 (2005).

11. Y.K. Choi, K. Sugimoto, S.M. Song, and M. Endo: Production and characterization of polycarbonate composite sheets reinforced with vapor grown carbon fiber. Composites Part A 37, 1944 (2006).

12. A. Eitan, F.T. Fisher, R. Andrews, L.C. Brinson, and L.S. Schadler: Reinforcement mechanisms in MWCNT-filled polycarbonate. Compos. Sci. Technol. 66, 1162 (2006).

13. B.K. Satapathy, R. Weidisch, P. Pötschke, and A. Janke: Toughto-brittle transition in multiwalled carbon nanotube (MWNT)/ polycarbonate nanocomposites. Compos. Sci. Technol. 67, 867 (2007).

14. F. Rouabah, M. Fois, L. Ibos, A. Boudenne, D. Dadache, N. Haddaoui, and P. Ausset: Mechanical and thermal properties of polycarbonate. II. Influence of titanium dioxide content and quenching on pigmented polycarbonate. J. Appl. Polym. Sci. 106, 2710 (2007).

15. L.E. Hornberger, G. Fan, and K.L. Devries: Effect of thermal treatment on the impact strength of polycarbonate. J. Appl. Phys. 60, 2678 (1986).

16. N.J. Mills: The mechanism of brittle-fracture in notched impact tests on polycarbonate. J. Mater. Sci. 11, 363 (1976).

17. J.T. Ryan: Impact and yield properties of polycarbonate as a function of strain rate, molecular weight, thermal history, and temperature. Polym. Eng. Sci. 18, 264 (1978).

18. C. Cheng, A. Hiltner, E. Baer, P.R. Soskey, and S.G. Mylonakis: Deformation of rubber-toughened polycarbonate: Macroscale analysis of the damage zone. J. Appl. Polym. Sci. 52, 177 (1994).

19. K-C. Ho, J-R. Hwang, and J-L. Doong: Impact fatigue of short glass fiber reinforced polycarbonate. J. Reinf. Plast. Compos. 16, 903 (1997).

20. G.L. Pitman, I.M. Ward, and R.A. Duckett: Effects of thermal pretreatment and molecular-weight on impact behavior of polycarbonate. J. Mater. Sci. 13, 2092 (1978).

21. S. Sarva, A.D. Mulliken, and M.C. Boyce: Mechanics of Taylor impact testing of polycarbonate. Int. J. Solids Struct. 44, 2381 (2007).

22. H.S. Shin, S.T. Park, S.J. Kim, J.H. Choi, and J.T. Kim: Deformation behavior of polymeric materials by Taylor impact. Int. J. Mod. Phys. B 22, 1235 (2008).

23. Y. Sato, M. Yoshida, K. Nagayama, and Y. Horie: Stress-strain relationships of polycarbonate over a wide range of strain rate, including a shock wave regime. Int. J. Impact Eng. 35, 1778 (2008).

24. V. Lerch, G. Gary, and P. Herve: Thermomechanical properties of polycarbonate under dynamic loading. J. Phys. IV (France) 110, 159 (2003).

25. A.D. Mulliken and M.C. Boyce: Polycarbonate and a polycarbonate-POSS nanocomposite at high rates of deformation. J. Eng. Mater. Technol. 128, 543 (2006).

26. S.S. Sarva and M.C. Boyce: Mechanics of polycarbonate during high-rate tension. J. Mech. Mater. Struct. 2, 1853 (2007).
27. Z. Xia, H-J. Sue, and A.J. Hsieh: Impact fracture behavior of molecularly orientated polycarbonate sheets. J. Appl. Polym. Sci. 79, 2060 (2001).

28. X. Hou and W. Goldsmith: Projectile perforation of moving plates: Experimental investigation. Int. J. Impact Eng. 18, 859 (1996).

29. R.P. Nimmer: An analytic study of tensile and puncture test behavior as a function of large-strain properties. Polym. Eng. Sci. 27, 263 (1987).

30. R.P. Nimmer: Analysis of the puncture of a bisphenol-a polycarbonate disc. Polym. Eng. Sci. 23, 155 (1983).

31. P. Pötschke, T.D. Fornes, and D.R. Paul: Rheological behavior of multiwalled carbon nanotube/polycarbonate composites. Polymer (Guildf.) 43, 3247 (2002).

32. O. Bunk, M. Bech, T.H. Jensen, R. Feidenhans'l, T. Binderup, A. Menzel, and F. Pfeiffer: Multimodal x-ray scatter imaging. N. J. Phys. 11, 123016 (2009).

33. T. Casiraghi, G. Castiglioni, and G. Ajroldi: A study of the impact behavior of injection molded polypropylene using 2 different modes of testing. Plast. Rubber Process. Appl. 2, 353 (1982).

34. Y. Duan, A. Saigal, R. Greif, and M.A. Zimmerman: Modeling multiaxial impact behavior of a glassy polymer. Mater. Res. Innovat. 7, 10 (2003).

35. T. Villmow, S. Pegel, P. Pötschke, and U. Wagenknecht: Influence of injection molding parameters on the electrical resistivity of polycarbonate filled with multi-walled carbon nanotubes. Compos. Sci. Technol. 68, 777 (2008).

36. G. Beaucage and D.W. Schaefer: Structural studies of complex systems using small-angle scattering: A unified Guinier powerlaw approach. J. Non-Cryst. Solids 172, 797 (1994).

37. R.J. Roe: Methods of X-ray and Neutron Scattering in Polymer Science (Oxford University Press, New York, 2000).

38. R.S. Justice, D.H. Wang, L-S. Tan, and D.W. Schaefer: Simplified tube form factor for analysis of small-angle scattering data from carbon nanotube filled systems. J. Appl. Cryst. 40, s88 (2007).

39. D.W. Schaefer and R.S. Justice: How nano are nanocomposites? Macromolecules 40, 8501 (2007).

40. D.W. Schaefer, T. Rieker, M. Agamalian, J.S. Lin, D. Fischer, S. Sukumaran, C.Y. Chen, G. Beaucage, C. Herd, and J. Ivie: Multilevel structure of reinforcing silica and carbon. J. Appl. Cryst. 33, 587 (2000).

41. T. Villmow, P. Pötschke, S. Pegel, L. Häussler, and B. Kretzschmar: Influence of twin-screw extrusion conditions on the dispersion of multi-walled carbon nanotubes in a poly(lactic acid) matrix. Polymer (Guildf.) 49, 3500 (2008).

42. M.C. Boyce and R.N. Haward: The post yield deformation of glassy polymers, in The Physics of Glassy Polymers, edited by R.N. Haward and R. Young (Chapman and Hall, London, UK, 1997), p. 213.

43. B.A.G. Schrauwen, L.C.A. van Breemen, A.B. Spoelstra, L.E. Govaert, G.W.M. Peters, and H.E.H. Meijer: Structure, deformation, and failure of flow-oriented semicrystalline polymers. Macromolecules 37, 8618 (2004).

44. S.C. Tjong and Y.Z. Meng: Effect of reactive compatibilizers on the mechanical properties of polycarbonate/poly(acrylonitrilebutadiene-styrene) blends. Eur. Polym. J. 36, 123 (2000).

45. W. Ding, A. Eitan, F.T. Fisher, X. Chen, D.A. Dikin, R. Andrews, L.C. Brinson, L.S. Schadler, and R.S. Ruoff: Direct observation of polymer sheathing in carbon nanotube-polycarbonate composites. Nano Lett. 3, 1593 (2003).

46. A.J. Hsieh, P. Moy, F.L. Beyer, P. Madison, E. Napadensky, J. Ren, and R. Krishnamoorti: Mechanical response and rheological properties of polycarbonate layered-silicate nanocomposites. Polym. Eng. Sci. 44, 825 (2004). 
47. B.K. Satapathy, M. Ganß, R. Weidisch, P. Pötschke, D. Jehnichen, T. Keller, and K.D. Jandt: Ductile-to-semiductile transition in PPMWNT nanocomposites. Macromol. Rapid Commun. 28, 834 (2007).

48. F.J. Carrión, J. Sanes, and M-D. Bermúdez: Influence of $\mathrm{ZnO}$ nanoparticle filler on the properties and wear resistance of polycarbonate. Wear 262, 1504 (2007).

49. M. Sánchez-Soto, D.A. Schiraldi, and S. Illescas: Study of the morphology and properties of melt-mixed polycarbonate-POSS nanocomposites. Eur. Polym. J. 45, 341 (2009).

50. T.D. Fornes, J.W. Baur, Y. Sabba, and E.L. Thomas: Morphology and properties of melt-spun polycarbonate fibers containing single- and multi-wall carbon nanotubes. Polymer (Guildf.) 47, 1704 (2006).

51. B. Hornbostel, P. Pötschke, J. Kotz, and S. Roth: Single-walled carbon nanotubes/polycarbonate composites: Basic electrical and mechanical properties. Phys. Status Solidi B 243, 3445 (2006).

52. W. Zhang, J. Suhr, and N.A. Koratkar: Observation of high buckling stability in carbon nanotube polymer composites. Adv. Mater. 18, 452 (2006).

53. R.W. Truss and T.K. Yeow: Effect of exfoliation and dispersion on the yield behavior of melt-compounded polyethylene- montmorillonite nanocomposites. J. Appl. Polym. Sci. 100, 3044 (2006).

54. N. Aït Hocine, P. Médéric, and T. Aubry: Mechanical properties of polyamide-12 layered silicate nanocomposites and their relations with structure. Polym. Test. 27, 330 (2008).

55. R.N. Rothon: Particulate-Filled Polymer Composites (Rapra Technology Limited, Shropshire, UK, 2003), pp. 377-419.

56. B. Chen and J.R.G. Evans: Impact and tensile energies of fracture in polymer-clay nanocomposites. Polymer (Guildf.) 49, 5113 (2008).

57. K. Prashantha, J. Soulestin, M.F. Lacrampe, P. Krawczak, G. Dupin, and M. Claes: Masterbatch-based multi-walled carbon nanotube filled polypropylene nanocomposites: Assessment of rheological and mechanical properties. Compos. Sci. Technol. 69, 1756 (2009).

58. Y. Dong and D. Bhattacharyya: Effects of clay type, clay/ compatibiliser content and matrix viscosity on the mechanical properties of polypropylene/organoclay nanocomposites. Composites Part A 39, 1177 (2008).

59. K.K. Kar, S. Srivastava, A. Rahaman, and S.K. Nayak: Acrylonitrile-butadiene-styrene nanocomposites filled with nanosized alumina. Polym. Compos. 29, 489 (2008). 